\title{
Effect of Following Physiotherapy on Strength and Range of Motion in Patients with Total Hip Arthroplasty: A Case Series
}

\author{
Shivangini Patel $^{1}$, Yiva Bhanushali ${ }^{2}$, Bhavana Gadhavi ${ }^{3}$ \\ ${ }^{1}$ MPT Scholar, ${ }^{2}$ BPT, Parul Institute of Physiotherapy, Parul University, Vadodara, Gujarat. \\ ${ }^{3}$ Dean and Principal at Parul Institute of Physiotherapy, Parul University, Vadodara, Gujarat.
}

Corresponding Author: Shivangini Patel

\begin{abstract}
Introduction: The hip is a true ball-and-socket joint surrounded by powerful and well-balanced muscles, enabling a wide range of motion in several physical planes while also exhibiting remarkable stability. The vascular supply to the femoral head has been well studied due to the risk of vascular necrosis of the head when it is disrupted, particularly in fractures of the femoral neck or dislocation of the hip. Total hip arthroplasty (THR) is the surgical procedure of choice, because this treatment improves the patient's quality of life and facilitates the patient's return to activities of daily living (ADLs) and even to labor activities.

Methodology: Total 5 patients with Total Hip Arthroplasty were taken. Patients who fulfill the inclusion criteria were included in the Study. Informed and written Consent were taken. Physiotherapy treatment was given in form of ROM Exercise and Strengthening Exercise Twice a day for 5 days.

Outcome Measures: Goniometer for ROM and MMT for Strength.

Result: Pre and post intervention data was recorded with Goniometer and MMT. Significance was assessed at 5\% level of significance. Significance improvement in ROM and Muscle strength of Hip, Knee and Ankle was noted.

Conclusion: Study shows significance effect of following Physiotherapy on Strength and ROM in patients with Total Hip Arthroplasty.
\end{abstract}

Keywords: Total Hip Arthroplasty, THA, Post-op. Physiotherapy

\section{INTRODUCTION}

The hip is a true ball-and-socket joint surrounded by powerful and wellbalanced muscles, enabling a wide range of motion in several physical planes while also exhibiting remarkable stability. As the structural link between the lower extremities and the axial skeleton, the hips not only transmit forces from the ground up but also carry forces from the trunk, head and neck, and upper extremities. Consequently this joint is crucial to athletic activities in which it is often exposed to many greater than normal axial and torsional forces. ${ }^{[1,2]}$ The hip joint is unique anatomically, physiologically, and developmentally; and therefore the diagnosis of pathologic conditions is more difficult than for most joints ${ }^{[3]}$. Because of these diagnostic challenges, the hip has received considerably less attention than other joints in the past, particularly in reference to sports medicine and surgery literature. The clinical setting of a plain $x$-ray of the pelvis exhibiting non-arthritic joints was a difficult situation - patients were potentially diagnosed erroneously with a 'groin strain' or otherwise. With the advent of improved magnetic resonance imaging (MRI) enhanced by arthrography, we now have a 
better comprehension of pathological processes within the hip joint. Accompanying this increased understanding is the evolving potential to treat these problems. For one, hip arthroscopy is undergoing continued development and excellent results have been reported treating a variety of intra-articular conditions ${ }^{[4,5]}$. While we can now assess and treat patients with newer diagnoses, we must also ensure that our knowledge of hip anatomy and biomechanics also evolves. Only with this fundamental understanding can the clinician or engineer provide adequate treatment for the patient suffering from hip disease or malfunction.

ANATOMY OF THE HIP: The hip is a classical ball-and-socket joint. It meets the four characteristics of a synovial or diarthrodial joint: it has a joint cavity; joint surfaces are covered with articular cartilage; it has a synovial membrane producing synovial fluid, and; it is surrounded by a ligamentous capsule. [6] For ease of approach we have considered the relevant anatomy under the headings 'Bony anatomy', 'Ligaments and capsular anatomy', 'Neurovascular anatomy' and 'Muscular anatomy'.

BONY ANATOMY: The cup-shaped acetabulum is formed by the innominate bone with contributions from the ilium (approximately $40 \%$ of the acetabulum), ischium $(40 \%)$ and the pubis $(20 \%)^{[7]}$. In the skeletally immature these three bones are separated by the triradiate cartilage fusion of this starts to occur around the age of $14-16$ years and is complete usually by the age of $23^{[8]}$. The actual articular surface appears a lunate shaped when viewed looking into the acetabulum. Within the lunate, or horseshoe shaped articular cartilage is a central area - the central inferior acetabular fossa. This fat filled space houses a synovial covered fat pad and also contains the acetabular attachment of the ligamentum teres. Inferior to this, the socket of the hip is completed by the inferior transverse ligament. Attached to the rim of the acetabulum is the fibrocartilaginous labrum. The labrum has been closely studied as tears of the labrum are the most common indication for hip arthroscopy ${ }^{[9]}$. Although it makes less of a contribution to joint stability than the glenoid labrum in the shoulder it does serve its purpose. It plays a role in normal joint development and in distribution of forces around the joint ${ }^{[10,11]}$. It has also been suggested it plays a role in restricting movement of synovial fluid to the peripheral compartment of the hip, thus helping exert a negative pressure effect within the hip joint [12]

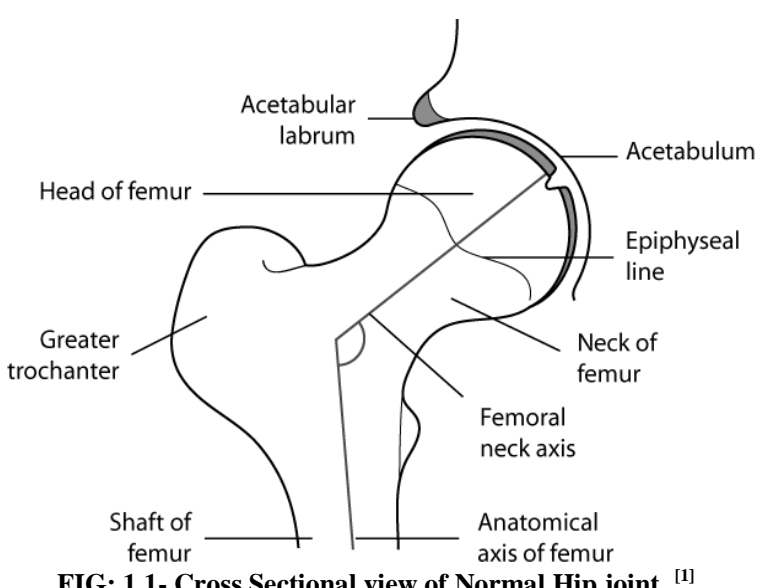

FIG: 1.1- Cross Sectional view of Normal Hip joint. ${ }^{[1]}$

The head of the femur is attached to the femoral shaft by the femoral neck, which varies in length depending on body size. The neck-shaft angle is usually $125 \pm 5^{\circ}$ in the normal adult, with coxa valga being the condition when this value exceeds $130^{\circ}$ and coxa vara when the inclination is less than 120. The importance of this feature is that the femoral shaft is laterally displaced from the pelvis, thus facilitating freedom for joint motion. If there is significant deviation in angle outside this typical range, the lever arms used to produce motion by the abductor muscles will either be too small or too large. The neck-shaft angle steadily decreases from $150^{\circ}$ after birth to $125^{\circ}$ in the adult due to remodelling of bone in response to changing stress patterns. The femoral neck in the average person is also rotated slightly anterior to the coronal plane. This medial rotation is referred to as femoral anteversion. The angle of 
anteversion is measured as the angle between a mediolateral line through the knee and a line through the femoral head and shaft. The average range for femoral anteversion is from 15 to $20^{\circ}$. The neck is most narrow midway down the neck. Abnormalities in this area and the area adjacent to the articular surface, such as a prominence resulting from a slipped capital femoral epiphysis (SCFE), can upset the normal femoroacetabular articulation leading to Cam type impingement. Conversely, abnormalities of the acetabulum such as osteophyte formation, with increased cover of the femoral head can lead to Pincer type impingement. The vascular supply to the femoral head has been well studied due to the risk of vascular necrosis of the head when it is disrupted, particularly in fractures of the femoral neck or dislocation of the hip. ${ }^{[1]}$
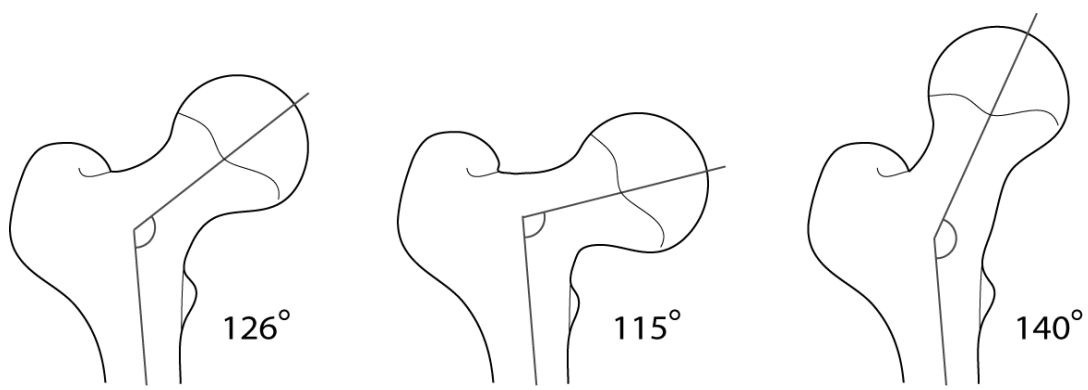

FIG: 1.2 Normal femoral neck angle, (b) a decreased femoral neck angle (coxa vara), and an increased femoral neck angle (coxa valga). ${ }^{[1]}$

Patients with a hip fracture are at substantial risk for death, health complications, and reduced quality of life. Despite the high frequency of the injury, the way in which displaced femoral neck fractures in elderly patients should be managed surgically remains uncertain. Options include or total hip arthroplasty, which involves replacement of both the femoral head and the acetabulum with prostheses. ${ }^{[13]}$. Total hip arthroplasty (THA) has completely revolutionized the nature in which the arthritic hip is treated, and is considered to be one of the most successful orthopaedic interventions of its generation. ${ }^{[14]}$ Total hip arthroplasty (THA) is considered to be one of the most successful orthopaedic interventions of its generation. The earliest recorded attempts at hip replacement occurred in Germany in 1891, with results presented at the 10th International Medical Conference. Professor Themistocles Glück presented the use of ivory to replace femoral heads of patients whose hip joints had been destroyed by tuberculosis. Later, surgeons experimented with interpositional arthroplasty in the late $19^{\text {th }}$ and early $20^{\text {th }}$ century, which involved placing various tissues (fascia lata, skin, pigbladders submucosa) between articulating hip surfaces of the arthritic hip. ${ }^{[14,15]}$ Total hip arthroplasty (THR) is the surgical procedure of choice, because this treatment improves the patient's quality of life and facilitates the patient's return to activities of daily living(ADLs)and even to labor activities. [15] Physiotherapy can improve strength and gait speed after THA and help prevent frequent complications, which include luxation and thromboembolic disease. In addition, physiotherapy increases the patient's mobility and offers education about the exercises and precautions that are necessary during hospitalization and after discharge. ${ }^{[16]}$ Reduce pain, swelling and stiffness and increased function have demonstrated in patients who received hydrotherapy after THA. ${ }^{[17]}$

Standard

physiotherapy rehabilitation programs for post THA patients are usually based on hip range of motion (ROM) exercises and functional activities typically without external loading and also fail to restore normal levels of muscle mass, strength and physical function in patients following elective THA for 
osteoarthritis. ${ }^{[18]}$ Structured rehabilitation program that included hydrotherapy sessions, daily hip and thigh exercises and regular walking or cycling, that is considerably more than most post THA rehabilitation regimes. ${ }^{[19]}$

\section{NEED OF STUDY}

Hip Arthroplasty is a surgical procedure done in Conditions like Intertrochanteric fractures, femoral neck fractures, Hip Dislocations, AVN of hip. Such conditions are at substantial risk of death so it can be treated with Hip Arthroplasty. After Surgery the Quality of Life get reduced as the ROM and strength of the limbs. Fractures is common problems in elderly patients who cannot perform heavy exercises or strength training exercises, but The Strength and ROM needs to improve to improve quality of life. There are many studies which show effect of advanced techniques on Hip Arthroplasty patients, but there are few studies which show the effect of Conventional Physiotherapy treatments in patients with Hip Arthroplasty.

So, here arrives the Need of study to find out the Effect of Following Physiotherapy on Strength and ROM in patients with Hip Arthroplasty.

\section{AIM AND OBJECTIVES OF STUDY AIM OF STUDY :-}

- To see Effect of Following Physiotherapy on Strength and ROM in patients with Hip Arthroplasty.

\section{OBJECTIVES OF STUDY:-}

- To see the Effect of Following Physiotherapy on Strength in patients with Hip Arthroplasty.

- To see the Effect of Following Physiotherapy on ROM in patients with Hip Arthroplasty.

\section{RESEARCH HYPOTHESIS: Null Hypothesis Ho}

H01 - There is no significant effect of Physiotherapy treatment on ROM in patients with Total Hip Arthroplasty.
H02 - There is no significant effect of Physiotherapy treatment on muscle strength in patients with Total Hip Arthroplasty.

\section{Alternative Hypothesis H1}

H11 - There is significant effect of Physiotherapy treatment on ROM in patients with Total Hip Arthroplasty.

H12-There is significant effect of Physiotherapy treatment on muscle Strength in patients with Total Hip Arthroplasty.

\section{REVIEW OF LITERATURE}

1. A study done by Catherine J Minns, Karen L Barker, and Catherine MSackley in year august 2009, titled "Effectiveness of physiotherapy exercise following hip arthroplasty for osteoarthritis: a systematic review of trials." Insufficient evidence exists to establish the effectiveness of physiotherapy exercise following primary hip replacement for osteoarthritis. Further well designed trials are required to determine the value of post discharge exercise following this increasingly common surgical procedure. This study indicates the physiotherapy exercise after discharge following total hip replacement has the potential to benefit patients.

2. A study done by AB Lemmy, T Okoro in year 2013 titled "The efficacy of exercise rehabilitation in restoring physical function following total hip replacement for osteoarthritis: a review." The studies reviewed suggest that centre-based, but not home based, exercise rehabilitation are effective in restoring muscle mass, strength and function in total hip arthroplasty patients during the immediate post-surgery phase, and that the efficacy of the centre-based interventions is most likely due to higher training intensity that is facilitated by supervision and access to specialised equipment and facilities. When commencement of training is delayed, however, both home- and centre-based training programs provide 
significant improvements in patient strength and function.

3. A study done by Felix Greimel, Gregor Dittrich, Timo Schwarz, Moritz Kaiser, Bernd Krieg, Florian Zeman, Joachim Grifka, Achim benditz in year 31 july 2018, titled "Course of pain after total hip arthroplasty within a standardized pain management concept: a prospective study examining influence, correlation, and outcome of postoperative pain on 103 consecutive patients." Increasing pain levels after the first week postoperatively, for 3 days, are most likely to be caused by the change to more extensive mobilization and physiotherapy in the rehabilitation unit. No significant influence or correlation on the intensity of postoperative pain could be found while evaluating potential predictors except preoperative pain levels. Pain management has to take these findings into account in the future to further increase patients' satisfaction in the postoperative course after total hip arthroplasty and to adapt pain management programs.

4. A study done by Annet Wijnen et al. PLos One in year 2018, titled "The Therapeutic Validity and Effectiveness of Physiotherapeutic Exercise Following Total Hip Arthroplasty for Osteoarthritis: A Systemic Review." The insufficient therapeutic validity and potentially high risk of bias in studies involving physiotherapeutic exercise interventions limit the ability to assess the effectiveness of these interventions following THA. Researchers are advised to take both scores into account when developing and reporting studies involving physiotherapeutic exercise. Uniformity in intervention characteristics and outcome measures in necessary to enhance the comparability of clinical outcomes between trials.

\section{MATERIALS \& METHODS}

STUDY DESIGN: Case series
STUDY POPULATION: Postoperative case of total Hip arthroplasty referred for physiotherapy.

STUDY DURATION: 3 Months

SOURCE OF DATA: Parul Sevashram hospital

SAMPLE SIZE: 5 patients

SAMPLING METHOD: Convenient Method

\section{INCLUSION CRITERIA}

1. Both gender

2. Subjects who have undergone Arthroplasty of the Hip.

\section{EXCLUSION CRITERIA}

1. Any complication following Hip Arthroplasty like Deep Vein Thrombosis, nerve injury.

2. Any Neurological deficit lower limb like stroke.

\section{OUTCOME MEASURE}

1. Hip, knee, and ankle Range of motion by Goniometry.

2. Muscle strength check by Manual Muscle testing.

\section{MATERIALS AND TOOLS}

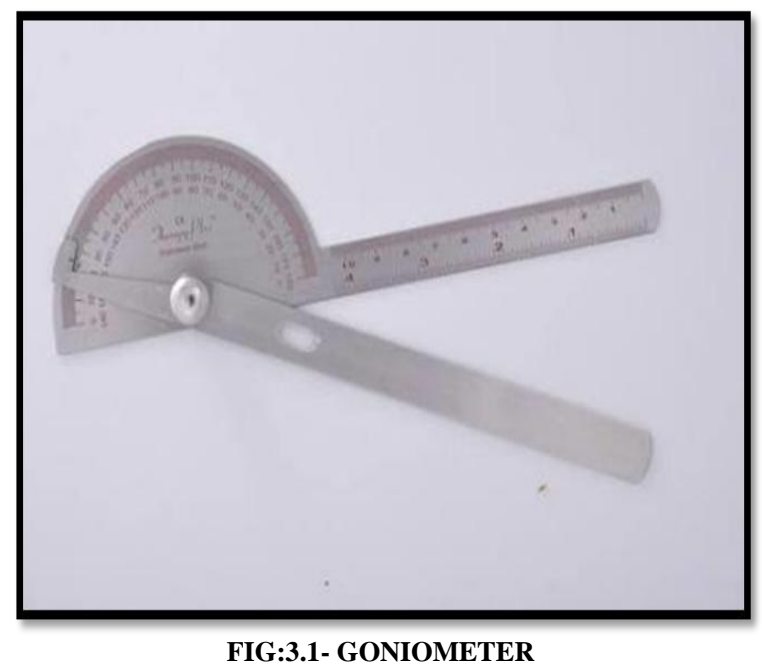

\section{PROCEDURE}

In the act of research consists of human subjects, ethical clearance was obtained from ethical committee of Parul Institutional Ethics committee for Human Research. Also written consent was taken 
from every subject who associates in study. After getting approval referred cases of Hip Arthroplasty from Parul Sevashram Hospital, fulfilling the inclusion criteria were included in the study. Patients were informed about the study procedure and informed consent was taken. Following that, Patients were assessed according to assessment format. Treatment was given 2 times a day

Range of motion exercise- Heel drag. Active Knee extension,

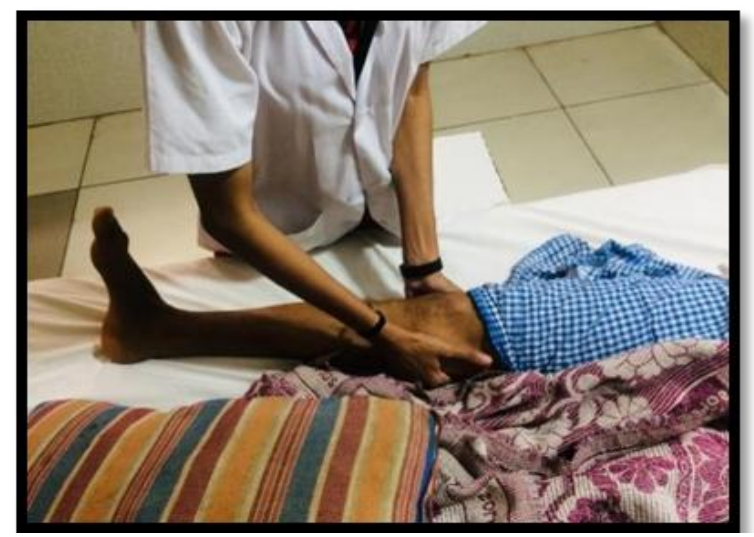

Fig:3.2- Static Quadriceps

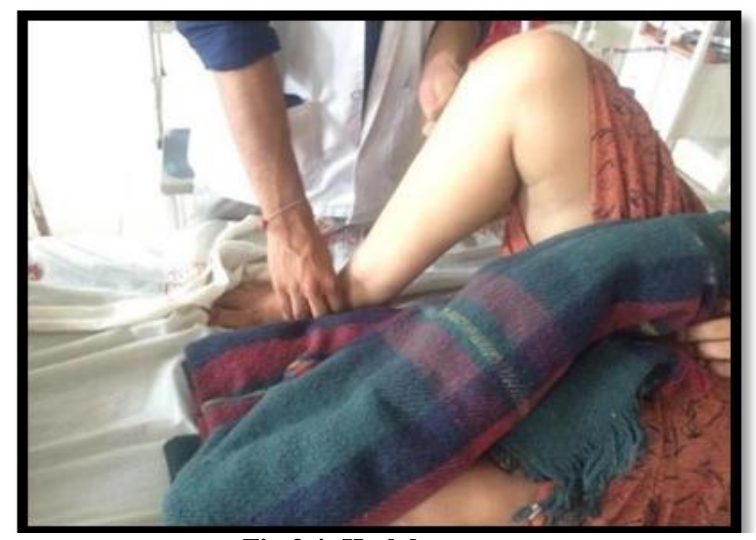

Fig:3.4- Heel drag
Strengthening exercise- Static abductors, Last degree knee extension, static quadriceps,

Treatment was given For 5 consecutive days in form of range of motion exercise and muscle strength exercise. Pre treatment and Post treatment outcome measures were taken in terms of Goniometry for hip and knee range and muscle strength on 1 stday and $5^{\text {th }}$ day. Data was collected and recorded in Microsoft Excel sheet.

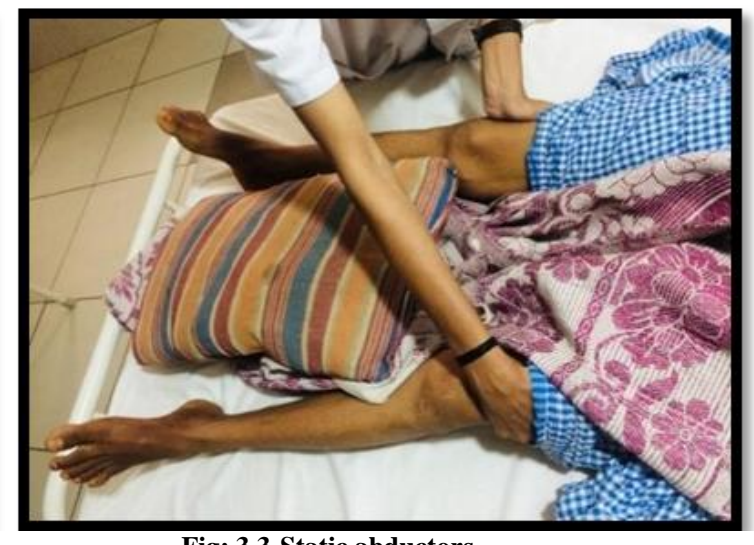

Fig: 3.3-Static abductors

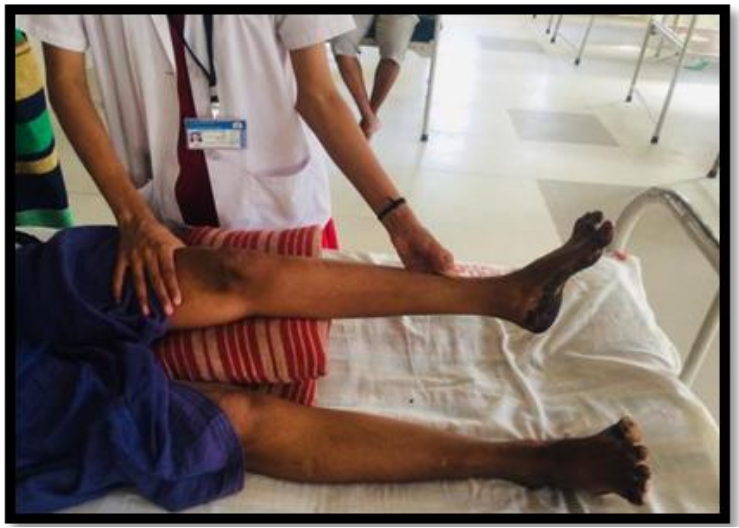

Fig:3.5- Last degree knee extension

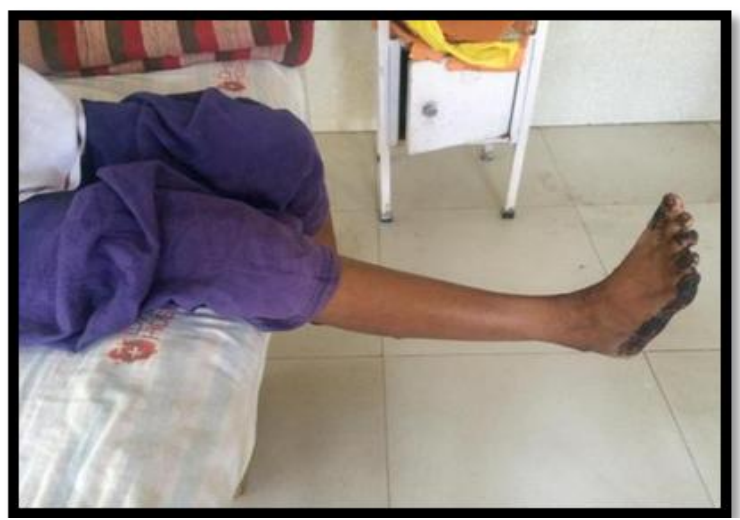

Fig:3.6-Knee extension (high sitting) 


\section{Statistical Analysis}

The data were collected and entered in excel sheet and results were analyzed by using statistical analysis.

\section{RESULT}

In the present study, 5 patients with Hip Arthroplasty were taken (2-Male, 3female). Pre and Post intervention record was taken. All participants completed the study program without any complications.

Microsoft Excel 2010 was used for the data analysis. Normality variant done and we found that data follows normality assumptions and therefore we have used Parametric test for Pre and Post intervention comparison of within group, paired t-test was used and we found Significance improvement in Hip Knee and Ankle ROM and Muscle strength. Data was analyzed at 95\% of Confidence interval.

\section{PRE and POST Comparison of ROM}

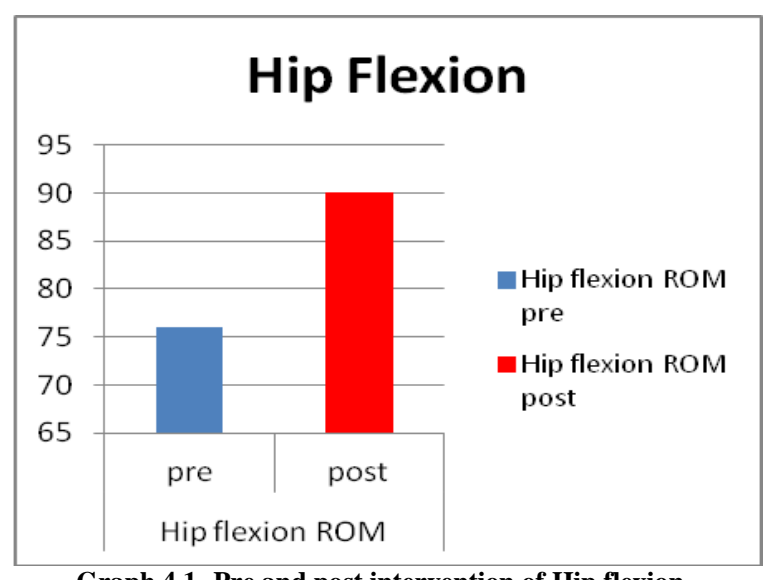

Graph 4.1- Pre and post intervention of Hip flexion

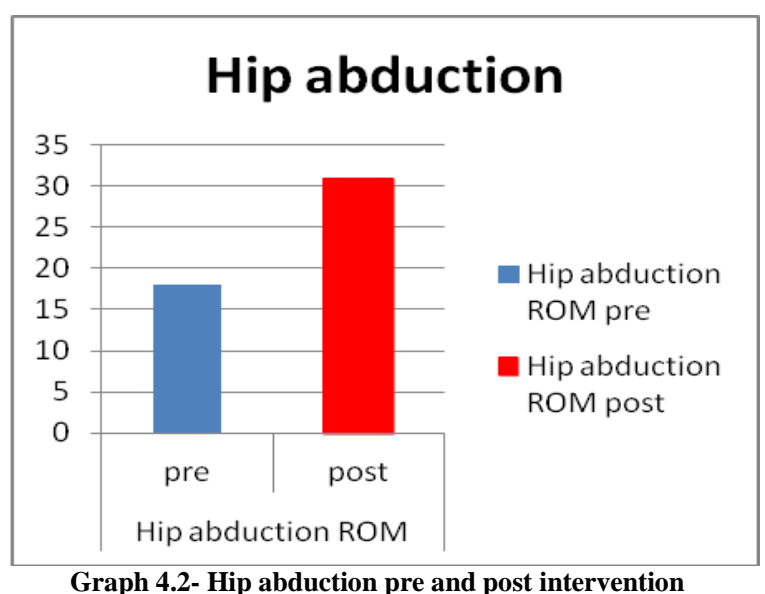

Graph 4.2- Hip abduction pre and post intervention
Graph 4.1 shows Pre and post mean difference of Intervention in Hip flexion ROM with pre Mean 76 and post mean 90.

Graph 4.2 shows Pre and post mean difference of Intervention in Hip Abduction ROM with pre Mean 18 and post mean 31.

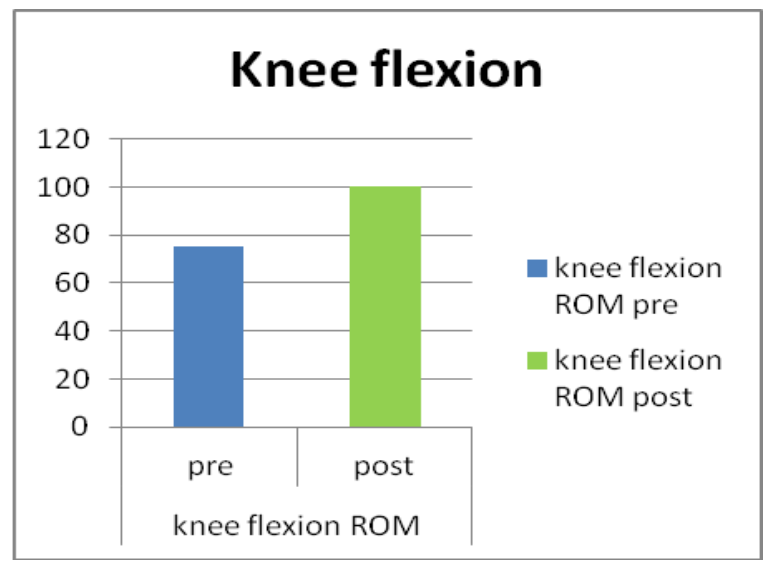

Fig 4.3- Knee flexion pre and post intervention.

Graph 4.3 shows Pre and post mean difference of Intervention in Knee flexion ROM with pre mean 75 and Post mean 100.

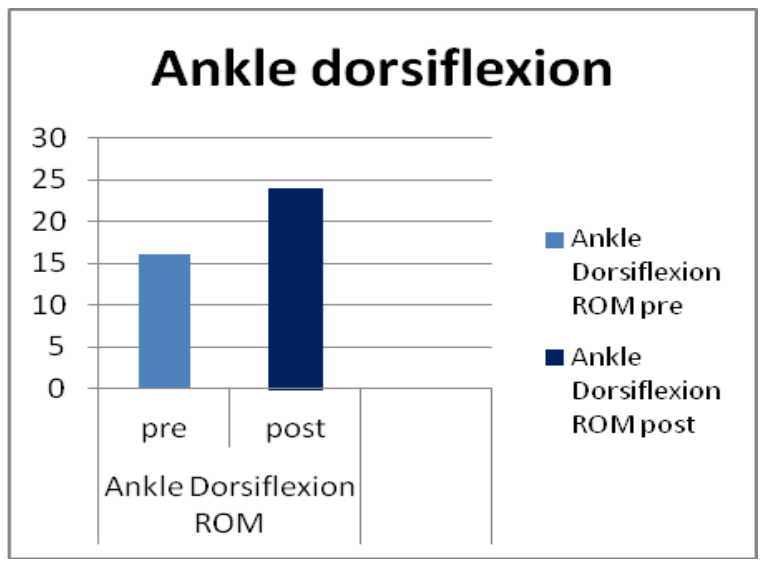

Fig 4.4- Ankle dorsiflexion pre and post intervention

\section{Ankle plantarflexion}

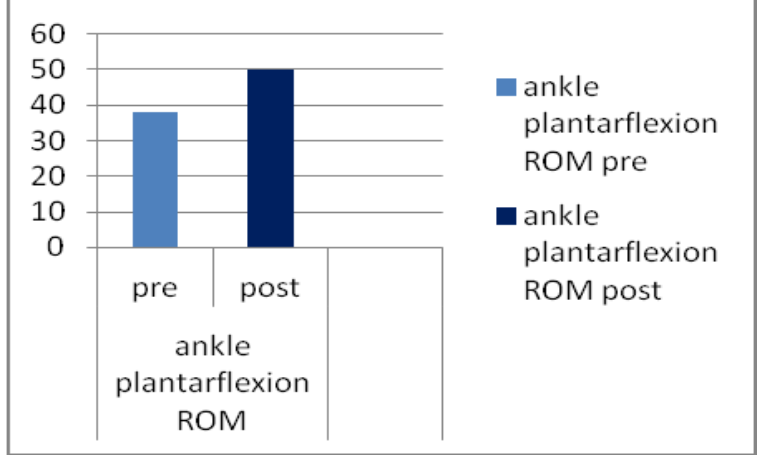

Fig: 4.5- Ankle plantarflexion pre and post intervention 
Graph 4.4 shows Pre and post mean difference of Intervention in Ankle dorsiflexion ROM with Pre Mean 16 and post mean 24.

Graph 4.5 shows Pre and post mean difference of Intervention in Ankle Plantarflexion ROM with Pre Mean 38 and Post Mean 50.

\section{PRE and POST Comparison of muscle strength by MMT}

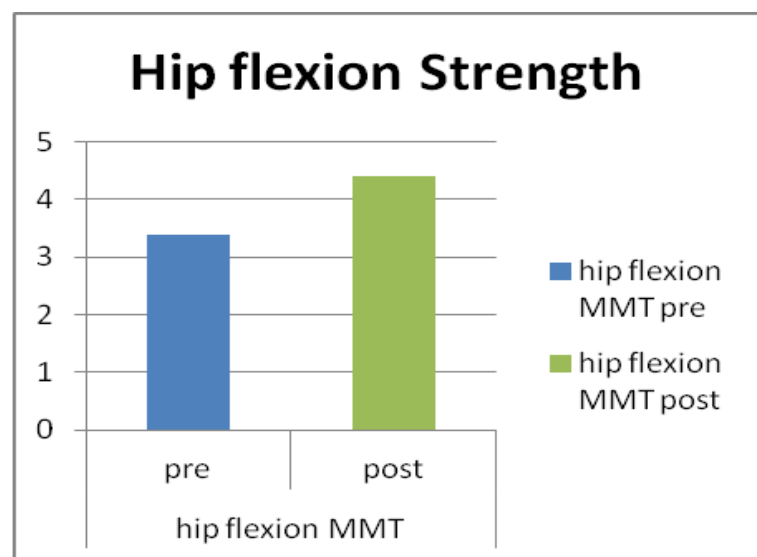

Fig 4.6- Pre and post intervention of Hip flexion muscle strength

Graph 4.6 shows Pre and post mean difference of Intervention in Hip flexion muscle strength with Pre Mean 3.4 and Post Mean 4.4.

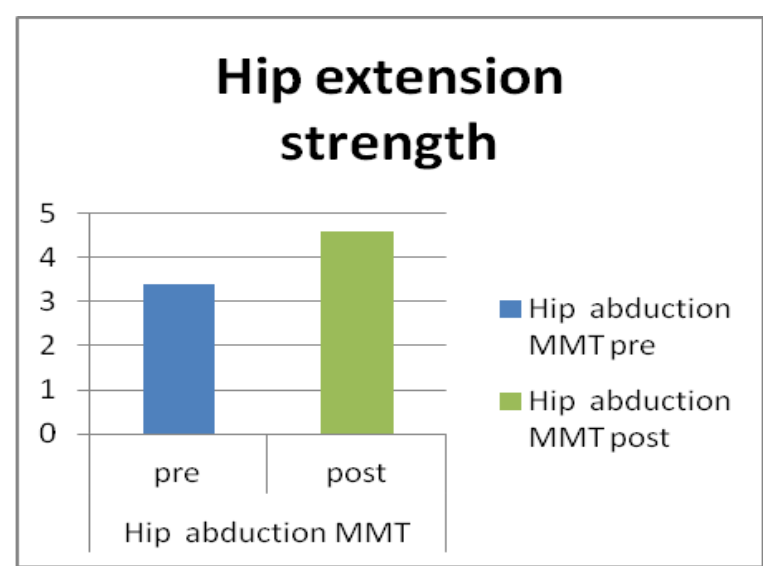

Graph 4.7- Pre and post intervention of Hip extension muscle strength.

Graph 4.7 shows Pre and post mean difference of Intervention in Hip Extension muscle strength with Pre Mean 3.4 and Post Mean 4.6

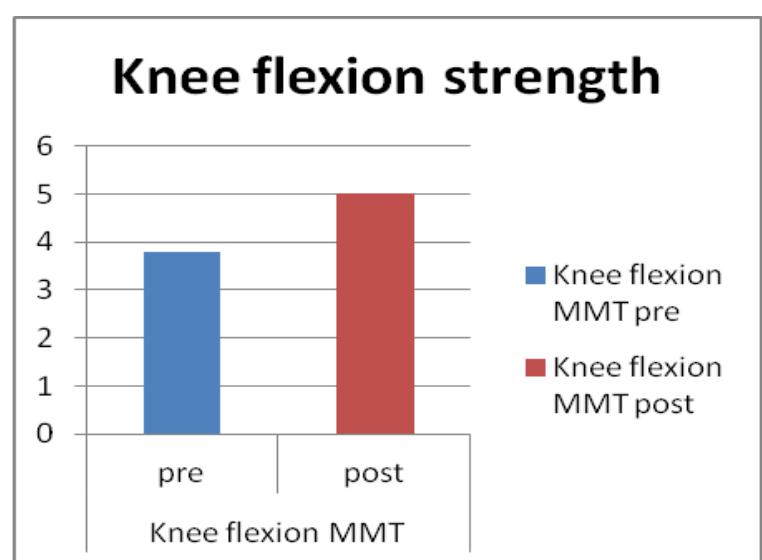

Graph 4.8- Pre and Post intervention of Knee flexion muscle strength

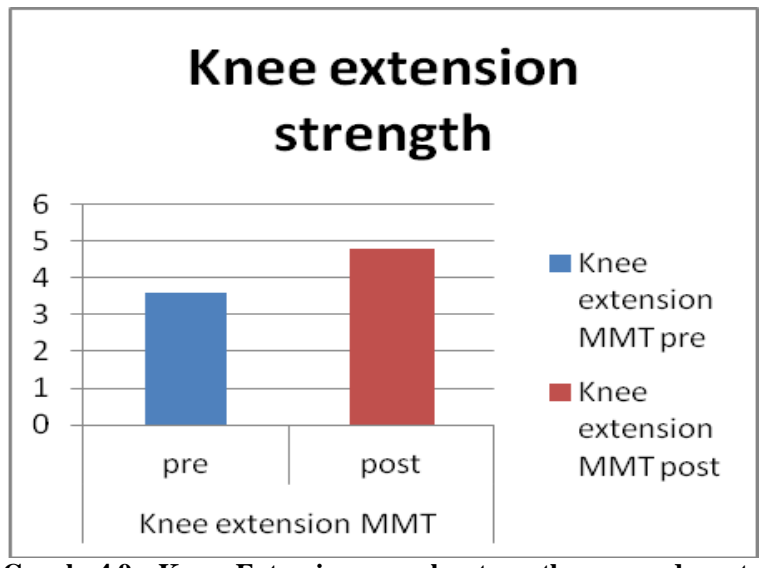

Graph 4.9- Knee Extension muscle strength pre and post intervention

Graph 4.8 shows Pre and post mean difference of Intervention in Knee flexion muscle strength with Pre Mean 3.8 and Post Mean 5.

Graph 4.9 shows Pre and post mean difference of Intervention in Knee extension muscle strength with Pre Mean 3.6 and Post Mean 4.8.

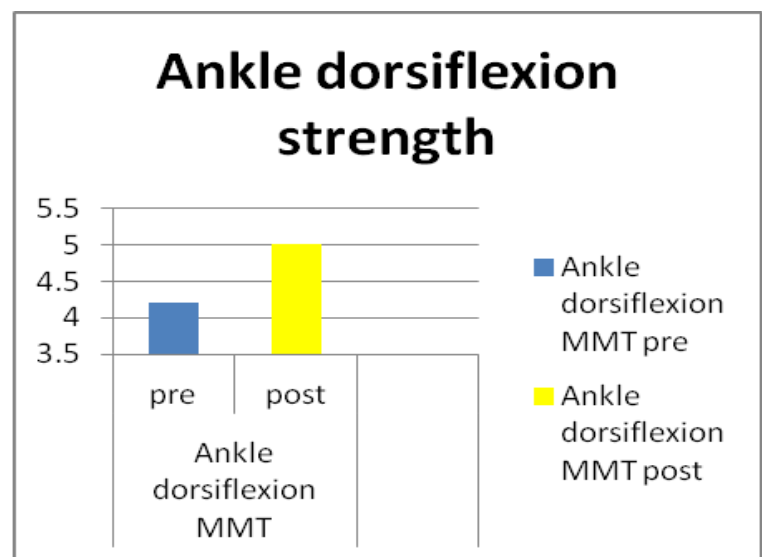

Graph 4.10- Pre and Post intervention of Ankle dorsiflexion muscle strength 


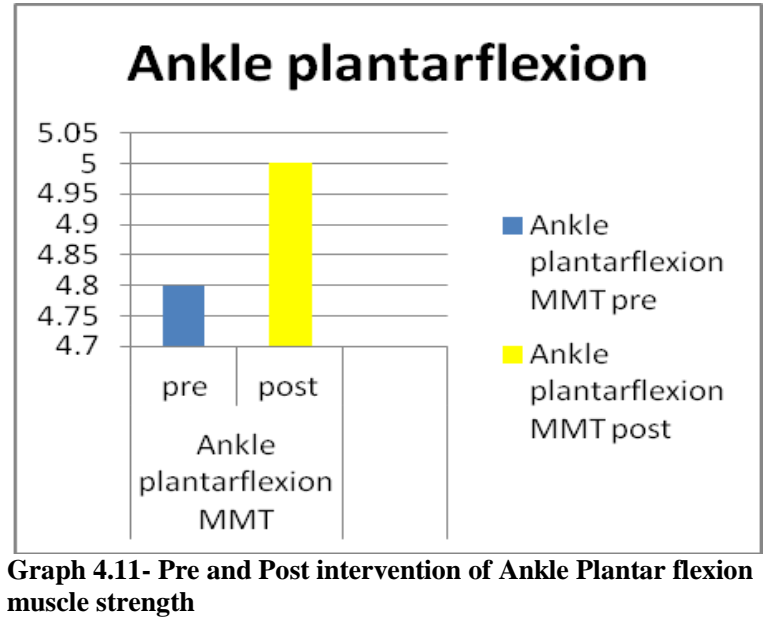

Graph 4.10 shows Pre and post mean difference of Intervention in Ankle Dorsiflexion muscle strength with Pre Mean 4.2 and Post Mean 5.

Graph 4.11 shows Pre and post mean difference of Intervention in Ankle Plantar flexion muscle strength with Pre Mean 4.8 and Post Mean 5.

\section{DISCUSSION}

In this study total 5 post operative total hip arthroplasty patients referred for physiotherapy were screened, out of which 5 were included in this study. Out of 5 patients, 2 patients were male and 3 was female.

Among 5 patients 4 patients were less than 40 years.

All patients' assessment and outcome were taken on post operative day 1 and 5 .

Treatment was given 2 times a day for 5 consecutive days.

The present study shows that range of motion and muscle strength was improved in all patients following physiotherapy treatment.

AB Lemmey, T Okoro had studied efficacy of exercise rehabilitation are effective in restoring muscle mass, strength and function in Total Hip Arthroplasty. Present study also shows that improvement in muscle strength.

Carolina Sant'anna Umpierres et al. had studied Rehabilitation following Total Hip Arthroplasty are effective in improving muscle strength force, range of motion and mobility.

\section{CONCLUSION}

The present study was aimed to check the effect of Following Physiotherapy on Range of Motion and Muscle Strength of Hip, Knee and Ankle in Patients undergone with Hip Arthroplasty. Based on the analysis and result it can be concluded that, Following Physiotherapy is effective in Improving Range of Motion and Muscle Strength of Hip knee and Ankle in Patients undergone with Hip Arthroplasty.

\section{SUMMARY}

Hip arthroplasty (THR) is the surgical procedure of choice, because this treatment improves the patient's quality of life and facilitates the patient's return to activities of daily living (ADLs) and even to labor activities. [15] Physiotherapy can improve strength and gait speed after THA and help prevent frequent complications, which include luxation and thromboembolic disease.

Total 5 patients with Hip Arthroplasty were taken from Parul Sevashram Hospital. Patients were selected according to inclusion and exclusion criteria. 5 subjects with Hip Arthroplasty were given Physiotherapy treatment in case to see effect on ROM and Muscle strength. Range of motion exercise in for of Heel drag and Active exercise of Hip knee and ankle was given and Strengthening exercise in form of Static quadriceps, Static abductors, Knee extension (high sitting) was given.

Baseline measurement of Goniometry and MMT was taken on 1st day and Post intervention data was taken after 5 days of treatment.

\section{Conclusion}

Following Physiotherapy is effective in Improving Range of Motion and Muscle Strength of Hip knee and Ankle in Patients undergone with Hip Arthroplasty. 


\section{Limitation of study}

- Study was done small sample size.

\section{Further recommendation}

- Further study can be done with larger sample size.

- Study can be done with Specific age group.

- Study can be done using advanced technique or treatments on Hip Arthroplasty patients.

\section{Acknowledgement: None}

\section{Conflict of Interest: None}

\section{Source of Funding: None}

Ethical Approval: Approved

\section{REFERENCES}

1. Damien P. Byrne, Kevin J. Mulhall and Joseph F. Baker. Anatomy \& Biomechanics of the Hip. The Open Sports Medicine Journal, 2010, 4, 51-57

2. Campbell JD, Higgs $R$, Wright $K$, Leaver-Dunn D. Pevis, hip and thigh injuries. In: Schenck RC, Guskiewicz KM, Holmes CF, Eds. Athletic Training and Sports Medicine. Rosemount: American Academy of Orthopaedic Surgeons 2001; p. 399

3. Mosca VS. Pitfalls in diagnosis: the hip. Pediatr Ann 1989; 18(1): 12-4, 16-8, 23.

4. McCarthy J, Barsoum W, Puri L, et al. The role of hip arthroscopy in the elite athlete. Clin Orthop Relat Res 2003; (406): 71-4.

5. Philippon M, Schenker M, Briggs K, Kuppersmith D. Femoroacetabular impingement in 45 professional athletes: associated pathologies and return to sport following arthroscopic decompression. Knee Surg Sports Traumatol Arthrosc 2007; 15(7): 90814.

6. Byrd J. Gross anatomy. In: Byrd J, Ed. Operative Hip Arthroscopy, 2nd ed. New York: Springer Science + Business Media, Inc. 2004; pp. 100-9.
7. Schuenke M, Schulte E, Schumacher U. THIEME Atlas of Anatomy. In: Ross L, Lamperti E, Eds. General Anatomy of the Musculoskeletal System. New York: Thieme New York 2006.

8. Moore K, Ed. Clinically Oriented Anatomy. 3rd ed. Baltimore: Williams and Wilkins 1992.

9. Byrd JWT. Indications and contraindications. In: Byrd JWT, Ed. Operative Hip Arthroscopy. 2nd ed. New York: Springer Science + Business Media, Inc. 2005.

10. Kim YH. Acetabular dysplasia and osteoarthritis developed by an eversion of the acetabular labrum. Clin Orthop Relat Res 1987; (215): 289-95.

11. Tanabe H. Aging process of the acetabular labrum--an electron microscopic study. Nippon Seikeigeka Gakkai Zasshi 1991; 65(1): 18-25.

12. Ferguson SJ, Bryant JT, Ganz R, Ito K. $\mathrm{An}$ in vitro investigation of the acetabular labral seal in hip joint mechanics. J Biomech 2003; 36(2): 1718.

13. Sheila Sprague, Ernesto Guerra, Frede Frihagen ,et al. Total Hip Arthroplasty or Hemiarthroplasty for Hip Fracture. New England Journal of Medicine September 2019.

14. Stephen Richard Knight,1 Randeep Aujla,2Satya Prasad Biswas. Total Hip Arthroplasty - over 100 years of operative history. Orthopaedics reviews 2011;3:e16.

15. Flugsrud GB, Nordslentten L, Reinholt FP, Risberg MA, Rydevik K, Uhlig T.[osteoarthritis].Tidsskrift for den Norske laegeforening: tidsskrift for praktisk medicin, ny raekke.Christiania og Kjobenhavn: Alb. Cammermeeyers Forlsg.2010;130(21);2136-40.

Norwegian.

16. Sharma L, Kapoor D, Issa S. Epidemiology of osteoarthritis:An update. Curr Opin Rheumatol. 2006; 18(2):142-56. 
17. Ritterman S, Rubin LE. Rehabiliation for total joint arthroplasty. R I Med J.2013;96(5):19-22.

18. National Joint Registry for England and Wales. 8th annual clinical report 2012 [cited 8 august 2013].

19. Rasch A, Bystrom AH, Dalen N, Martinez-Carranza N, Berg $\mathrm{H}$. Persisting muscle atrophy two years after replacement of the hip. J Bone Joint Surg Br.2009 May;91(5):583-8.

How to cite this article: Patel S, Bhanushali Y, Gadhavi B. Effect of following physiotherapy on strength and range of motion in patients with total hip arthroplasty: a case series. Int J Health Sci Res. 2021; 11(4): 122-132. DOI: https:// doi.org/10.52403/ijhsr.20210417

$* * * * * *$ 